\title{
A healthy delivery of twins by assisted reproduction followed by preimplantation genetic screening in a woman with X-linked dominant incontinentia pigmenti
}

\author{
Myung Joo Kim', Sang Woo Lyu', Hyun Ha Seok', Ji Eun Park², Sung Han Shim²*, Tae Ki Yoon* \\ 'Department of Obstetrics and Gynecology, ${ }^{2}$ Genetics Laboratory, Fertility Center of CHA Gangnam Medical Center, CHA University College of \\ Medicine, Seoul, Korea
}

\begin{abstract}
The purpose of this study is to report a successful twin pregnancy and delivery in a female patient with X-linked dominant incontinentia pigmenti (IP) who underwent assisted reproductive technology followed by preimplantation genetic screening (PGS). A 29-year-old female with IP had a previous history of recurrent spontaneous abortion. A molecular analysis revealed the patient had a de novo mutation, 1308_1309insCCCCTTG(p.Ala438ProfsTer26), in the inhibitor of the kappa B kinase gamma gene located in the Xq28 region. IVF/ICSI and PGS was performed, in which male embryos were sexed using array-based comparative genomic hybridization (aCGH). After IVF/ICSI and PGS using aCGH on seven embryos, two euploid male blastocysts were transferred with a $50 \%$ probability of a viable male pregnancy. The dizygotic twin pregnancy was confirmed and the amniocentesis results of each twin were normal with regard to the mutation found in the mother. The patient delivered healthy twin babies during the 37th week of gestation. This case shows the beneficial role of PGS in achieving a successful pregnancy through euploid male embryo gender selection in a woman with X-linked dominant IP with a history of multiple male miscarriages.
\end{abstract}

Keywords: Assisted reproductive technology; Gene mutation; Preimplantation screening; Recurrent miscarriage; X chromosome

Received: Oct 25, 2014 · Revised: Dec 1, 2014 · Accepted: Dec 4, 2014

Co-corresponding authors: Sung Han Shim

Genetics Laboratory, Fertility Center of CHA Gangnam Medical Center,

566 Nonhyeon-ro, Gangnam-gu, Seoul 135-913, Korea

Tel: +82-2-3468-3000 Fax:+82-2-3468-2610 E-mail:shshim@cha.ac.kr

\section{Tae Ki Yoon}

Department of Obstetrics and Gynecology, Fertility Center of CHA Gangnam

Medical Center, 566 Nonhyeon-ro, Gangnam-gu, Seoul 135-913, Korea

Tel: +82-2-3468-3000 Fax:+82-2-3468-2610 E-mail:tkyoon@cha.ac.kr

*These authors contribute equally to this study.

*This research was supported by the Basic Science Research Program through the National Research Foundation of Korea (NRF) funded by the Ministry of Education (2009-0093821).

This is an Open Access article distributed under the terms of the Creative Commons Attribution Non-Commercial License (http://creativecommons.org/licenses/by-nc/3.0/) which permits unrestricted non-commercial use, distribution, and reproduction in any medium, provided the original work is properly cited.

\section{Introduction}

Incontinentia pigmenti (IP), also known as Bloch-Sulzberger disease, is a rare $X$-linked dominant genodermatosis that affects varying parts of the body, including the dermatological, ocular, nervous, and immune systems, and is generally lethal in male fetuses $[1,2]$. IP is caused by mutations in the inhibitor of kappa $B$ kinase gamma (IKB$K G)$ gene, also known as the nuclear factor-kappa B essential modulator gene, in the Xq28 region. The most common mutations (60\%$80 \%$ of cases) involve a large-scale deletion of IKBKG exons 4 through 10 [3-5].

Though IP is related to recurrent spontaneous abortions in male fetal pregnancies, there have been few reports of attempts to achieve successful pregnancies in patients with this condition through assisted reproductive technology and genetic testing techniques. In the 
past, patients with IP underwent in vitro fertilization and male embryo selection using preimplantation genetic diagnosis (PGD) with fluorescent in situ hybridization (FISH), resulting in either a normal male pregnancy or early miscarriage in the case of affected male fetuses while excluding possible female carriers [6-8]. However, no cases of successful childbirth resulting from this approach have yet been reported. Recent case reports of PGD in patients with IP have included molecular analyses of the IKBKG gene through polar body biopsies, but this method does not involve a comprehensive genomic analysis $[9,10]$.

We report a successful pregnancy and delivery in a female patient with IP who underwent in vitro fertilization/intracytoplasmic sperm injection and preimplantation genetic screening (PGS) using arraybased comparative genomic hybridization $(\mathrm{aCGH})$ followed by a prenatal diagnosis.

\section{Case report}

A 29-year-old patient with known IP was referred to Fertility Center of CHA Gangnam Medical Center for fertility therapy. She had manifested skin erythema followed by vesicles as a neonate. Patchy hyperpigmented skin lesions subsequently appeared in multiple sites. At the time of her first visit to our institution, she had only mild skin lesions involving scattered hyperpigmented spots in the abdomen and upper legs. She had experienced three early spontaneous pregnancy losses during three years of marriage. No anatomical, immunological, thrombophilic or endocrinological factors contributed to recurrent spontaneous abortions in this patient. She had been clinically diagnosed with IP by Landy and Donnai's criteria [11] and by skin biopsy at ten years of age in the dermatology department of the referring hospital, with no family history. A cytogenetic analysis of this couple revealed normal karyotypes, but confirmatory molecular genetic analysis of IP had not been completed when assisted reproductive technology was applied. After genetic counseling, she was scheduled for an IVF cycle with PGS using aCGH to select euploid male embryos. Simultaneously, mutation screening was performed using polymerase chain reaction-direct sequencing to identify her pathogenic mutation, which is crucial for further genetic counseling and prenatal diagnosis. Genomic DNA was extracted from a blood sample. All exons and intron boundaries of the IKBKG gene were analyzed and a pathogenic mutation was identified in IKBKG exon 9 (1308_1309insCCCCTTG(p.Ala438ProfsTer26)). A seven-base insertion of CCCCTTG at position c.1309 was identified, which had resulted in a frameshift in which the 438th amino acid was changed from alanine to proline and a premature stop codon occurred at the 464th codon (Figure 1).

\section{IKBKG/NEMO}

\section{OMIM: *300248/ GenBank accession number: NM_001099856.3}

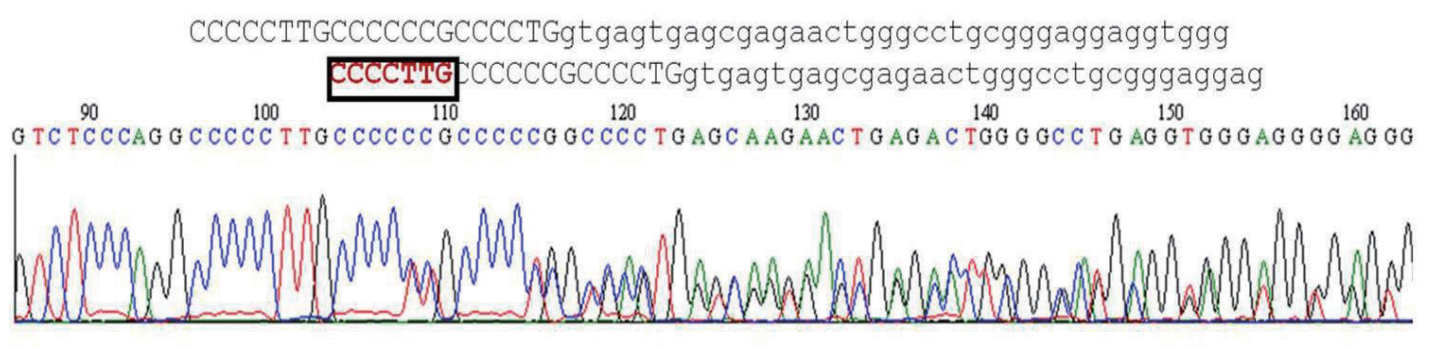

p.Ala438ProfsTer26

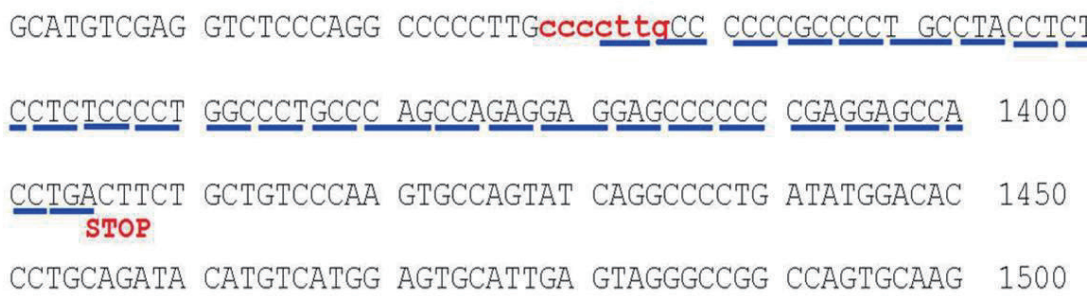

Figure 1. Polymerase chain reaction-direct sequencing in the IKBKG gene of the patient: a pathogenic mutation is present in IKBKG exon 9(1308_1309insCCCCTTG(p.Ala438ProfsTer26)), in which the seven-base sequence CCCCTTG was inserted at position c.1309, resulting in a frameshift (the 438th amino acid changed from alanine to proline) and a premature stop codon at the 464th codon. NEMO, nuclear factor-kappa B (NF-KB) essential modulator; IKBKG, inhibitor of KB kinase gamma; PB1, first polar body. 
A total of nine oocytes were retrieved after controlled ovarian hyperstimulation with gonadotrophin-releasing hormone antagonist protocol (cetrorelix, Cetrotide, Merck Serono Europe Ltd., London, UK; $0.25 \mathrm{mg}$ ) using recombinant follicle-stimulating hormone (recombinant follitropin alfa; GONAL-f, Merck Serono S.p.A, Modugno, Italy, 225 IU daily), of which seven were fertilized using the intracytoplasmic sperm injection procedure. In each of the seven eight-cell stage embryos, a single blastomere was biopsied on day three and subjected to aCGH analysis by a commercial laboratory (MGMED Co., Seoul, Korea). The laboratory reported that there were two euploid male embryos, one euploid female embryo, and four aneuploid male embryos $(-16,-8 /+15,+20,+2 /+3 /+6 /+11 /-20)$ (Figure 2). Both of the euploid male embryos, which were grade two mid-blastocysts, were transferred on day five. After 12 days, serum beta human chorionic gonadotropin was $442.17 \mathrm{mlU} / \mathrm{mL}$, increasing to $4,676 \mathrm{mlU} / \mathrm{mL}$ six days later. A clinical pregnancy was confirmed with two gestational sacs and twin fetuses showing viable heartbeats by ultrasonography at the sixth week of gestation. The patient underwent amniocentesis for a confirmatory prenatal diagnosis during the seventeenth week of pregnancy and both fetuses showed normal male karyotypes. They were shown to be normal for the mutation found in the mother through polymerase chain reaction-direct sequencing of the cultured amniocytes. This patient delivered healthy live twin babies during the 37 th week of gestation with no obstetric or neonatal complications.

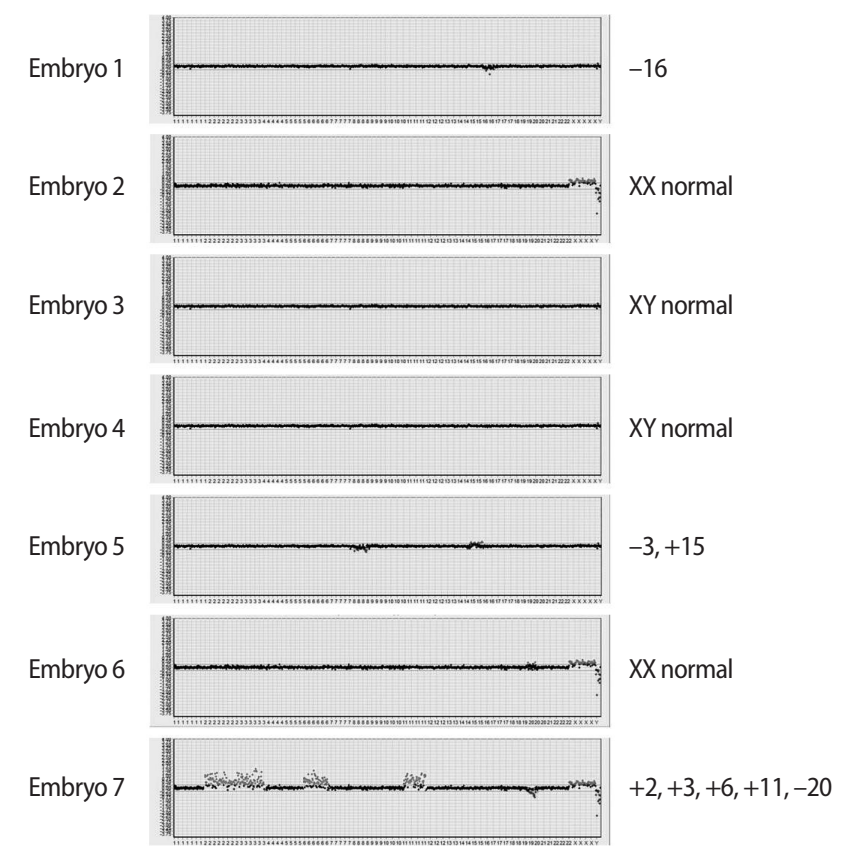

Figure 2. Array-based comparative genomic hybridization results of the seven embryos.

\section{Discussion}

IP is a rare X-linked dominant genodermatosis with its incidence of $0.7-2 / 100,000$ newborns [12-14], which is lethal in males in utero in $97 \%$ of cases [15]. As a result, many women with IP have recurrent early miscarriages [16]. Although IP is usually lethal in males, approximately 72 cases of surviving male fetuses with IP have so far been reported [17]. The IP Consortium has proposed three mechanisms resulting in the survival of males carrying a mutation in the IKBKG gene: hypomorphic alleles, the 47,XXY karyotype (Klinefelter syndrome), and somatic mosaicism [16]. IP presents multisystemically but especially dermatologically, involving four typical stages of vesiculo-bullous, verrucous, hyperpigmented, and hypopigmented skin. Diagnostic criteria for IP have traditionally been based on the clinical features established by Landy and Donnai [11]. Several researchers have recently proposed that these criteria should be updated to reflect a firmer molecular understanding of how the nuclear factor-kB pathway is affected by the IKBKG gene mutation $[18,19]$. Approximately $65 \%$ of those mutations occur de novo and 69 different mutations have been reported [5,20,21].

The patient in this study had a phenotypically very mild form of IP that only manifested dermatologically. Her family history, including her parents and one brother, showed no evidence of IP. She had a de novo insertion/deletion (indel) mutation causing a frameshift and a premature stop codon, which occurs in $10 \%$ of IP cases. Her mutation in IKBKG exon 9, 1308_1309insCCCCTTG(p.Ala438ProfsTer26), is the first reported genetic aberration affecting the IKBKG gene. This finding is analogous to other mutations, which have also been reported just once [21].

Many cases of recurrent spontaneous abortions in patients with IP have been reported, reflecting its $\mathrm{X}$-linked dominant nature and lethality to male fetuses, resulting in the suggestion that PGD should be used for patients with IP [22]. However, only a few cases have been reported of PGD used in embryos from IP-affected females in infertile couples who underwent IVF procedures (Table 1) [6,8-10]. FISH methods have conventionally been used to sex male embryos in the course of $P G D$ in patients with $I P$, which entails the possibility of either viable male fetal pregnancies or miscarriages if affected male embryos are transferred $[6,8]$. However, in all of these case reports using FISH methods with fewer than six chromosome probes, the fetuses were spontaneously aborted before confirmatory prenatal diagnosis resulting from chromosomal aneuploidies which were not tested.

In two recent case reports, the PGD for patients with IP was performed through polar body analysis strategies using polymerase chain reaction primers of the IKBKG gene to focus on the molecular basis of IP. The authors reported the delivery of a healthy boy and an ongoing pregnancy with one male fetus after 32 weeks of gestation 


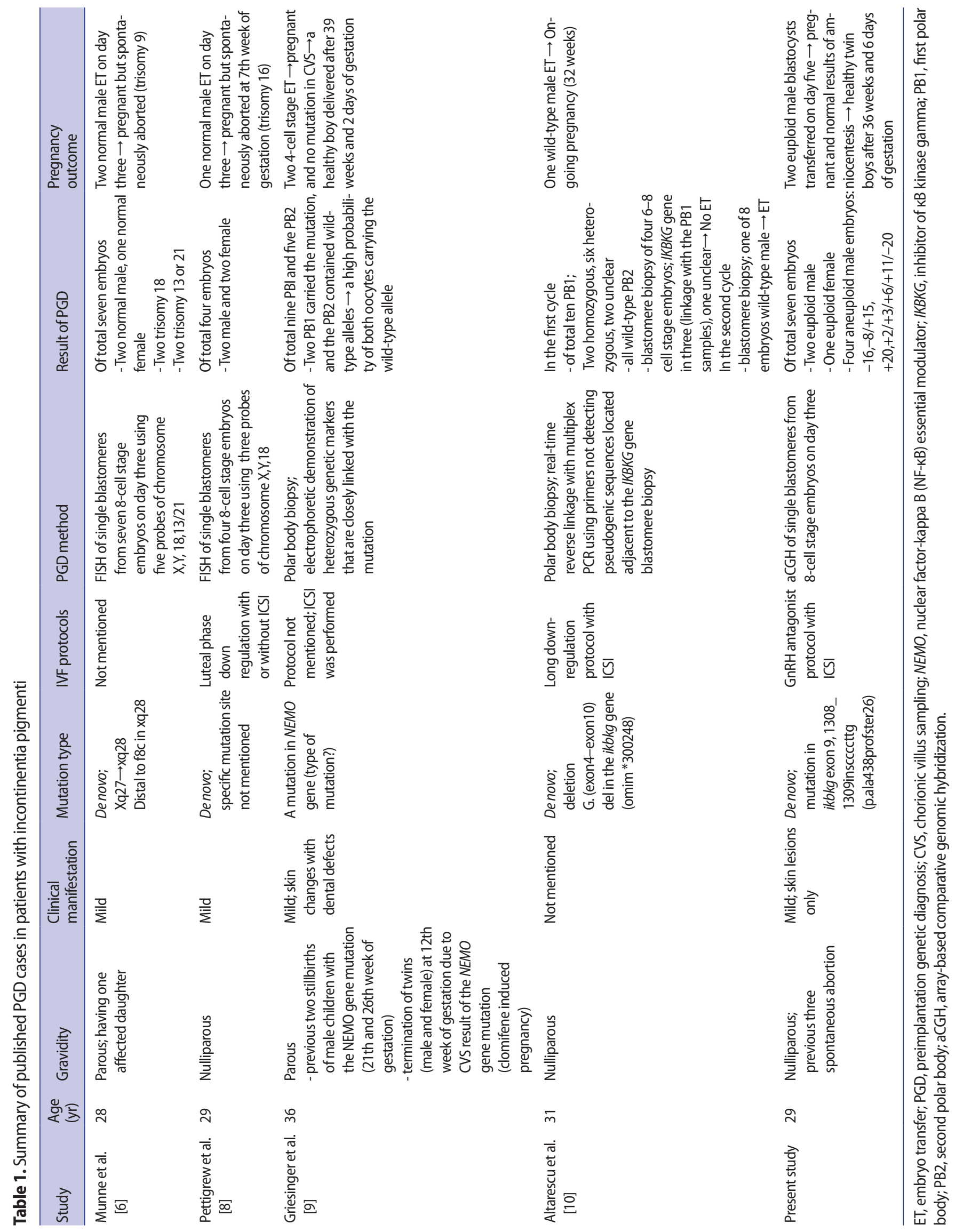


$[9,10]$. In a more recent study evaluating 151 PGD cycles using polar body analysis for de novo mutations in 38 different genetic disorders, the researchers applied eight PGD cycles to five patients with IP. They reported four births from eight embryos, which were transferred after sequential polar body analysis and embryo karyotyping of blastomeres for several chromosomes by polymerase chain reaction [23].

In this case, we also selected male embryos with a 50\% probability of a viable male pregnancy. However, we performed PGS as part of the embryo transfer process followed by amniocentesis with a molecular analysis of possible IP after the clinical confirmation of pregnancy. The patient delivered healthy dizygotic twin babies at the 37th week of gestation. We were able to prevent a miscarriage arising from an aneuploid pregnancy involving other autosomal mutations through a whole chromosome analysis using aCGH instead of only sexing the embryo, which was a significant difference from previous studies.

Although successful pregnancy outcomes have been presented in recent case reports in which polar body analysis was used, these reports have mainly focused on the molecular analysis of possible mutations inherited from the mother, without performing whole genome analysis. It is well known that aneuploidies are common in early human embryos $[24,25]$. According to a study that used FISH probes for chromosomes $X, Y, 13,15,16,17,18,21$, and 22 in 6054 cleavagestage embryos [26], 70\% of embryos showed chromosomal abnormalities. In another study analyzing aCGH of 70 single blastomeres, the authors reported that $55.7 \%$ of the blastomeres were diploid, $44.3 \%$ contained chromosomal abnormalities, and $29 \%$ were abnormal cells with structural aberrations [25]. The relatively high aneuploidy rate of $57 \%$ previously reported in IP cases [6] corresponds to the aneuploidy found in four of the seven embryos in our case. In light of this, embryo selection in IP patients through comprehensive genomic analytical techniques like aCGH, as performed in this study, is a promising approach.

Recently, next-generation sequencing has emerged as a PGS strategy both for mutation target diagnosis and simultaneous whole genome analysis $[27,28]$. Another very promising study has been published about single-gene disorders like IP, but the clinical applicability of the relevant technology remains to be confirmed [29].

In conclusion, this case shows the beneficial role of PGS in achieving successful pregnancy through sexing euploid embryos in a woman with X-linked dominant IP who had experienced multiple male miscarriages.

\section{Conflict of interest}

No potential conflict of interest relevant to this article was reported.

\section{References}

1. Hadj-Rabia S, Rimella A, Smahi A, Fraitag S, Hamel-Teillac D, Bonnefont JP, et al. Clinical and histologic features of incontinentia pigmenti in adults with nuclear factor-kappaB essential modulator gene mutations. J Am Acad Dermatol 2011;64:508-15.

2. Speeckaert R, Van Gele M, Speeckaert MM, Lambert J, van Geel N. The biology of hyperpigmentation syndromes. Pigment Cell Melanoma Res 2014;27:512-24.

3. Smahi A, Courtois G, Vabres P, Yamaoka S, Heuertz S, Munnich A, et al. Genomic rearrangement in NEMO impairs NF-kappaB activation and is a cause of incontinentia pigmenti. The International Incontinentia Pigmenti (IP) Consortium. Nature 2000;405:46672.

4. Fusco F, Pescatore A, Bal E, Ghoul A, Paciolla M, Lioi MB, et al. Alterations of the IKBKG locus and diseases: an update and a report of 13 novel mutations. Hum Mutat 2008;29:595-604.

5. Fusco F, Pescatore A, Steffann J, Royer G, Bonnefont JP, Ursini MV. Clinical Utility Gene Card for: incontinentia pigmenti. Eur J Hum Genet. 2013;21. doi: 10.1038/ejhg.2012.227.

6. Munne S, Alonso ML, Grifo J. Case report: unusually high rates of aneuploid embryos in a 28-year old woman with incontinentia pigmenti. Cytogenet Cell Genet 1996;72:43-5.

7. McGrath JA, Handyside AH. Preimplantation genetic diagnosis of severe inherited skin diseases. Exp Dermatol 1998;7:65-72.

8. Pettigrew R, Kuo HC, Scriven P, Rowell P, Pal K, Handyside A, et al. A pregnancy following $\mathrm{PGD}$ for X-linked dominant [correction of X-linked autosomal dominant] incontinentia pigmenti (BlochSulzberger syndrome): case report. Hum Reprod 2000;15:2650-2.

9. Griesinger G, Bundgen N, Salmen D, Schwinger E, Gillessen-Kaesbach $\mathrm{G}$, Diedrich $\mathrm{K}$. Polar body biopsy in the diagnosis of monogenic diseases: the birth of three healthy children. Dtsch Arztebl Int 2009;106:533-8.

10. Altarescu G, Eldar-Geva T, Varshower I, Brooks B, Haran EZ, Margalioth $\mathrm{EJ}$, et al. Real-time reverse linkage using polar body analysis for preimplantation genetic diagnosis in female carriers of de novo mutations. Hum Reprod 2009;24:3225-9.

11. Landy SJ, Donnai D. Incontinentia pigmenti (Bloch-Sulzberger syndrome). J Med Genet 1993;30:53-9.

12. Nelson DL. NEMO, NFkappaB signaling and incontinentia pigmenti. Curr Opin Genet Dev 2006;16:282-8.

13. Fryssira H, Kakourou T, Valari M, Stefanaki K, Amenta S, Kanavakis E. Incontinentia pigmenti revisited. A novel nonsense mutation of the IKBKG gene. Acta Paediatr 2011;100:128-33.

14. Orphanet report series. Prevalence of rare diseases: bibliographic data [Internet]. Paris: Orphanet; 2014 [cited 2014 Sep 1]. Available from:// http://www.orpha.net/orphacom/cahiers/docs/GB/ 
Prevalence_of_rare_diseases_by_alphabetical_list.pdf.

15. Ardelean D, Pope E. Incontinentia pigmenti in boys: a series and review of the literature. Pediatr Dermatol 2006;23:523-7.

16. Kenwrick S, Woffendin H, Jakins T, Shuttleworth SG, Mayer E, Greenhalgh $L$, et al. Survival of male patients with incontinentia pigmenti carrying a lethal mutation can be explained by somatic mosaicism or Klinefelter syndrome. Am J Hum Genet 2001;69: 1210-7.

17. Buinauskaite E, Buinauskiene J, Kucinskiene V, Strazdiene D, Valiukeviciene $S$. Incontinentia pigmenti in a male infant with Klinefelter syndrome: a case report and review of the literature. Pediatr Dermatol 2010;27:492-5.

18. Berlin AL, Paller AS, Chan LS. Incontinentia pigmenti: a review and update on the molecular basis of pathophysiology. J Am Acad Dermatol 2002;47:169-87.

19. Minic S, Trpinac D, Obradovic M. Incontinentia pigmenti diagnostic criteria update. Clin Genet 2014;85:536-42.

20. Fusco F, Paciolla M, Napolitano F, Pescatore A, D'Addario I, Bal E, et al. Genomic architecture at the Incontinentia Pigmenti locus favours de novo pathological alleles through different mechanisms. Hum Mol Genet 2012;21:1260-71.

21. Conte MI, Pescatore A, Paciolla M, Esposito E, Miano MG, Lioi MB, et al. Insight into IKBKG/NEMO locus: report of new mutations and complex genomic rearrangements leading to incontinentia pigmenti disease. Hum Mutat 2014;35:165-77.

22. Gigarel N, Frydman N, Burlet P, Kerbrat V, Steffann J, Frydman R, et al. Single cell co-amplification of polymorphic markers for the indirect preimplantation genetic diagnosis of hemophilia $A, X$ - linked adrenoleukodystrophy, X-linked hydrocephalus and incontinentia pigmenti loci on Xq28. Hum Genet 2004;114:298-305.

23. Rechitsky S, Pomerantseva E, PakhalchukT, Pauling D, Verlinsky $\mathrm{O}$, Kuliev A. First systematic experience of preimplantation genetic diagnosis for de-novo mutations. Reprod Biomed Online 2011;22: 350-61.

24. Munne S, Cohen J. Chromosome abnormalities in human embryos. Hum Reprod Update 1998;4:842-55.

25. Mertzanidou A, Wilton L, Cheng J, Spits C, Vanneste E, Moreau Y, et al. Microarray analysis reveals abnormal chromosomal complements in over $70 \%$ of 14 normally developing human embryos. Hum Reprod 2013;28:256-64.

26. Munne S, Chen S, Colls P, Garrisi J, Zheng X, Cekleniak N, et al. Maternal age, morphology, development and chromosome abnormalities in over 6000 cleavage-stage embryos. Reprod Biomed Online 2007;14:628-34.

27. Martin J, Cervero A, Mir P, Martinez-Conejero JA, Pellicer A, Simon $C$. The impact of next-generation sequencing technology on preimplantation genetic diagnosis and screening. Fertil Steril 2013;99:1054-61.e3.

28. Fiorentino F, Biricik A, Bono S, Spizzichino L, Cotroneo E, Cottone G, et al. Development and validation of a next-generation sequencing-based protocol for 24-chromosome aneuploidy screening of embryos. Fertil Steril 2014;101:1375-82.

29. Treff NR, Fedick A, Tao X, Devkota B, Taylor D, Scott RT Jr. Evaluation of targeted next-generation sequencing-based preimplantation genetic diagnosis of monogenic disease. Fertil Steril 2013; 99:1377-84.e6. 